${ }^{2}$ University of New South Wales, Sydney, NSW, Australia; and ${ }^{3}$ Department of Surgery, Prince of Wales Hospital, NSW Health Pathology East, Sydney, NSW, Australia

Dermatofibrosarcoma protuberans (DFSP) is a slow growing malignant tumour usually identified at the dermal/subcutaneous junction with a tendency for recurrence after incomplete resection, however metastatic disease is rare. In addition to the classic monotonous storiform arrangement of spindled cells, a number of histologic variants have been identified including myxoid, pigmented, and plaque-like variants. Fibrosarcomatous variants have been described which represent high grade transformation of the DFSP with acquisition of metastatic potential and these tumours have an increased tendency for recurrence. These are distinguished from the lower grade myoid variant.

We present a case of a 25 -year-old male who presented with a right groin mass. Microscopically the majority of the tumour showed the characteristic cytomorphologic features of a dermatofibrosarcoma protuberans with mild to moderately pleomorphic spindled cells arranged in a well developed storiform pattern with CD34 positivity. Within the storiform areas there was a striking perivascular proliferation of myoid spindle cells. In other areas away from vessels, the myoid spindle cells were arranged in fascicles, raising the possibility of fibrosarcomatous transformation, but these lacked obvious nuclear atypia or increased mitotic activity. The myoid appearing cells were focally positive for SMA and negative for CD34.

Dermatofibrosarcoma protuberans with areas of myoid differentiation do not appear to behave more aggressively than conventional DFSP and it is therefore important to distinguish them from the fibrosarcomatous variant.

\section{OESOPHAGEAL INVOLVEMENT IN PEMPHIGUS VULGARIS: A CASE REPORT}

Arfa Nawazish $^{1}$, Yancey Wilson ${ }^{1}$, Jean Iacobelli ${ }^{1}$, Lloyd D'Orsogna ${ }^{2}$

${ }^{1}$ Department of Anatomical Pathology, PathWest Laboratory Medicine, QE II Medical Centre, WA, Australia; and

${ }^{2}$ Department of Immunology, PathWest Laboratory Medicine, Fiona Stanley Hospital, WA, Australia

Pemphigus vulgaris is an autoimmune blistering disorder characterised by skin and mucosal involvement and an incidence of $0.5-3.2$ per 100,000 . It affects men and women with equal frequency, most commonly during 4 th and 5 th decade of life. It is a potentially life threatening disease, with a mortality rate of $5-15 \%$, attributed to the disease itself as well as complications secondary to corticosteroids and immunosuppressive therapy. Oesophageal involvement is rare and usually presents with dysphagia and odynophagia. Even when present, it may be under-recognised or misdiagnosed without a proper endoscopic and histopathologic evaluation.

We present a case of 76-year-old gentleman who developed swollen gums and severe oral ulceration which was associated with pain and difficulty swallowing along with significant loss of weight. He was admitted to hospital and required intravenous fluids and nasogastric tube feeding given substantial dehydration and malnutrition. He went onto develop flaccid blisters on abdomen and upper back. Skin biopsy was performed and histopathology supplemented with direct immunofluorescence (DIF) confirmed pemphigus vulgaris. The patient was commenced on prednisolone and was planned to undergo whole body PET scan, upper GI endoscopy and colonoscopy to rule out paraneoplastic pemphigus. The esophago-gastroscopy revealed multiple ulcers that were biopsied and histopathology showed suprabasal blister formation and acantholysis compatible with the diagnosis of pemphigus vulgaris.

\section{AN ATYPICAL PRESENTATION OF GIANT CELL ARTERITIS INVOLVING THE FACIAL ARTERY}

William Hao Syuen $\mathrm{Ng}^{1}$, Lisa Fiorentino ${ }^{2}$

${ }^{1}$ University of Newcastle, NSW, Australia; and ${ }^{2}$ Department of Anatomical Pathology, John Hunter Hospital, NSW, Australia

Giant cell arteritis (GCA) is a large vessel vasculitis that characteristically involves the temporal arteries. Rarely, it may involve other arteries with varying manifestations. We present a case of atypical location for GCA - the facial artery.

To date, there are only several case reports of GCA with facial artery involvement with no larger-scale studies. Our patient initially presented with $2-3$ weeks of bilateral jaw pain, submandibular swelling, lethargy, and oral paraesthesia. Following admission, she had visual changes diagnosed as transient ischaemic neuropathy, indicative of GCA. Her past medical history included polymyalgia rheumatica diagnosed 13 months prior. Her inflammatory markers (CRP, ESR and ferritin) were elevated. CT scans and MRI did not reveal any pathology, however on vascular ultrasound, a section of her left facial artery showed 'halo sign', increased thickness of the arterial walls and decreased compressibility; this section was subsequently biopsied.

Histology showed the presence of multinucleated giant cells which would be consistent with GCA. Also present was extensive disruption and fragmentation of the internal elastic lamina. There was an inflammatory infiltrate within the arterial wall, with lymphocytes, plasma cells, and histiocytes present. The tunica intima was also hyperplastic, and in some sections thrombosis of the artery was present.

\section{CD34-NEGATIVE SOLITARY FIBROUS TUMOUR OF THE BROAD LIGAMENT WITH DOEGE-POTTER SYNDROME: A CASE REPORT}

\section{Dan Nguyen, Katrina Tang}

Department of Anatomical Pathology, Prince of Wales Hospital, Sydney, NSW, Australia

Solitary fibrous tumours (SFT) are rare fibroblastic mesenchymal tumours that can occur in various anatomical sites but are extremely rare in the female genital tract. The broad ligament itself is a rare location for primary tumours and SFT in the broad ligament has only been described twice before in the literature. We report on a case of CD34-negative SFT in a 61-year-old 
female who presented with Doege-Potter syndrome. This case highlights the unusual presentation and location, atypical CD34 expression and utility of STAT6 immunohistochemistry for SFTs.

\section{References}

1. Chen S, Zheng Y, Chen L, et al. A broad ligament solitary fibrous tumor with Doege-Potter syndrome. Medicine 2018; 97: e12564.

2. Zubor P, Kajo K, Szunyogh N, et al. A solitary fibrous tumor in the broad ligament of the uterus. Pathol Res Pract 2007; 203: 555-60.

3. Ronchi A, Cozzolino I, Marino FZ, et al. Extrapleural solitary fibrous tumor: A distinct entity from pleural solitary fibrous tumor. An update on clinical, molecular and diagnostic features. Ann Diagn Pathol 2018; 34: 142-50.

\section{HUNGRY FOR KNOWLEDGE: EXPLORING PATHOLOGY ALIMENTARY ANALOGIES THROUGH CULINARY CREATIONS}

Minh Anh Nguyen, Esther Myint

Department of Anatomical Pathology, Douglass Hanly Moir

Pathology, Sydney, NSW, Australia

Objectives: To explore alimentary analogies in pathology through culinary creations and discuss their implications for pathologists and trainees.

Background: Alimentary analogies abound in the history and current practice of pathology, being a valuable tool in the diagnostic process. They can serve as a useful learning tool to recognise and remember pathological patterns and disease entities. ${ }^{1,2}$ They are an effective way of communicating pathological findings, by adding flavour to macroscopic and microscopic descriptions.

Method: A literature search was performed for commonly used alimentary analogies in pathology. Recipes for 'sago spleen' (amyloidosis), ovarian 'chocolate cyst' (endometriotic cyst) and 'anchovy paste' (amoebic abscess) liver and placenta (derived from Greek, meaning 'flat cake') were created.

Results: A literature search yielded 96 medical alimentary analogies. ${ }^{1-3}$ Culinary creations resembling pathology specimens, based on selected alimentary analogies were successfully produced, sampled, consumed and digested by staff at our institution.

Conclusions: Alimentary analogies in pathology can be further explored through culinary creations, which not only serve to satisfy the pathologist's hunger for knowledge, but also as a valuable learning and communication tool in the diagnostic process.

\section{References}

1. Masukume G, Zumla A. Analogies and metaphors in clinical medicine. Clin Med 2012; 12: 55-6.

2. Pena GP, Andrade-Filho JS. Analogies in medicine: valuable for learning, reasoning, remembering and naming. Adv Health Sci Educ Theory Pract 2010; 15: 609-19.

3. Vimal M, Nishanthi A. Food eponyms in pathology. J Clin Diagn Res 2017; 11: EE01-6.

\section{A CASE OF SPONTANEOUS INTESTINAL PERFORATION ASSOCIATED WITH CANDIDIASIS IN A PREMATURE INFANT}

\author{
C. Noy, H. Harris
}

Department of Anatomical Pathology, Royal Hobart Hospital, Hobart, Tas, Australia

Intestinal perforation is a potentially life-threatening complication of pre-term birth. Focal or spontaneous intestinal perforation (SIP) is a type of intestinal perforation occurring in extreme prematurity which is less common than, and is reported to demonstrate some clinically and histologically distinct features from, necrotising enterocolitis (NEC). This case report describes a case of SIP of the small bowel associated with candidiasis in an extremely premature infant girl. Histologically, the bowel wall lacked the extensive coagulative necrosis typically seen in NEC; rather, the adjacent bowel showed some possible attenuation of the muscularis propria and a serosal fibrinoinflammatory exudate, with the mucosa and remainder of the bowel wall otherwise well preserved. Fungal spores and scattered pseudpohyphael elements within the serosal exudate and, very focally, within the outer half of the muscularis propria were only identified histologically after performance of special stains on the resection specimen. An association between SIP and candidial sepsis has previously been reported in the literature and this case highlights that identification of such cases can be aided by performing special stains on cases with clinical and/or histological features in keeping with SIP.

\section{OVARIAN DLBCL PRESENTING AS A VERY LARGE OVARIAN MASS IN A YOUNG WOMAN - A CASE REPORT (AND POTENTIAL FROZEN SECTION PITFALL)}

\section{Noy, P. Jessup}

Department of Anatomical Pathology, Royal Hobart Hospital, Hobart, Tas, Australia

While ovarian involvement as a late manifestation of disseminated non-Hodgkin lymphoma (NHL) is not uncommon, primary ovarian NHL, or NHL presenting for the first time as an ovarian mass, is rare, having a reported incidence of around $0.5 \%$ of all untreated NHL and $1.5 \%$ of untreated ovarian neoplasms. This case study reports a 27-year-old woman who underwent a unilateral salpingo-ophorectomy and staging for a very large, rapidly enlarging, right ovarian mass, suspected clinically of being a dysgerminoma. On frozen section, the tumour was favoured to be a germ cell tumour, with juvenile granulosa cell tumour also not excluded. On permanent section, however, the tumour more clearly demonstrated morphological features and immunological features in keeping with a large B-cell nonHodgkin lymphoma. Coupled with FISH analysis showing no evidence of a MYC gene rearrangement, a diagnosis of DLBCL, NOS, double expressor phenotype, was ultimately made.

This case serves as a reminder that, although rare as an initial presentation in the ovary, lymphoma should be considered by 